\title{
Recursive graphs with small-world scale-free properties
}

\author{
Francesc Comellas \\ Departament de Matemàtica Aplicada IV, EPSC, Universitat Politècnica de Catalunya \\ Avinguda del Canal Olímpic s/n, 08860 Castelldefels, Barcelona, Catalonia, Spain * \\ Guillaume Fertin \\ IRIN, Université de Nantes, 2 rue de la Houssinière, BP 92208, 44322 Nantes Cedex 3, France 0 \\ André Raspaud \\ LaBRI, Université Bordeaux I, 351 Cours de la Libération, 33405 Talence Cedex, France团 \\ (Dated: Rec. 26 November 2002; revised manuscript rec. 15 December 2003; publ. 31 March 2004)

\begin{abstract}
We discuss a category of graphs, recursive clique trees, which have small-world and scale-free properties and allow a fine tuning of the clustering and the power-law exponent of their discrete degree distribution. We determine relevant characteristics of those graphs: the diameter, degree distribution, and clustering parameter. The graphs have also an interesting recursive property, and generalize recent constructions with fixed degree distributions.
\end{abstract} \\ PACS numbers: 89.75.Hc, 89.75.Da, 89.20.Hh
}

In a complex system a large number of agents interact showing a cooperative behavior which does not depend only on the individual features of its parts, but also on its structure and on the different sort of relations which can be established between them and the environment. This global behavior allows the system to attain certain achievements without the presence of an administrative hierarchy or a central control mechanism. A swarm of bees, an ant colony, companies which supply a big city (water, electricity, telephone, etc.), even biological mechanisms or social relationships are all complex systems where global patterns emerge from the interaction of a large number of similar elements. In recent years, there has been an increase in understanding of such complex systems in terms of networks, modeled by graphs composed of vertices and edges, where vertices represent the basic elements and edges their interactions. The World Wide Web (WWW), Internet, transportation systems, and many biological and social systems have been characterized by small-world scale-free networks [1, 2] which have a strong local clustering (nodes have many mutual neighbors), a small diameter (maximum distance between any two nodes) and a distribution of the number of nearest neighbors for each node according to a power law (scale-free property), see, for example, Refs. 3, 4, [5, 6]. To model these networks and their growth very often stochastic models and methods from statistical physics have been considered; see Ref. 7]. However, the use of exact deterministic models allows a quick determination of the relevant parameters of the associated graph that

*URL: http://wWw-mat.upc.es/ ${ }^{\sim}$ comellas Electronic address: comellas@mat.upc.es

†URL: http://www.sciences.univ-nantes.fr/info/perso/permanent Electronic address: fertin@irin.univ-nantes.fr

${ }^{\ddagger}$ URL: http://www.labri.fr/Perso/ ${ }^{\sim}$ raspaud/ Electronic address: raspaud@labri.fr may be compared with experimental data from real and simulated networks. Previous work, for example, considered deterministic small-world networks comparable to those obtained stochastically by Watts and Strogatz [8], node replacement and node addition methods to produce small-world and scale-free networks from a low diameter "backbone" network [9], specific recursive scale-free constructions with fixed degree distributions 10, 11, 12, 13. and scale-free trees (without clustering) [14]. In this paper we present a recursive graph construction which produces scale-free small-world networks with an adjustable clustering and such that the parameter of the power law associated with the degree distribution, $\gamma$, takes values between 2 and $1+\ln 3 / \ln 2=2.58496$. It has been shown that the values for the scaling exponent of most real technical networks [15] are in this range. Moreover, the addition of one-degree nodes to our construction allows a model which is not far from the real topology of Internet at the autonomous system level, as is described in Ref. [16].

Therefore, we introduce here a deterministic exact network model of complex systems for which we can adjust both the clustering parameter and the power-law exponent, providing a complementary tool to stochastic approaches.

Recursive clique tree $K(q, t), q \geq 2$. Definition.- a complete graph $K_{q}$ (also referred in the literature as $q$ clique; see Ref. 17]) is a graph without loops whose $q$ vertices are pairwise adjacent. The recursive clique tree $K(q, t)$ is the graph constructed as follows: For $t=0$, $K(q, 0)$ is the complete graph $K_{q}$ (or $q$ clique). For $t \geq 1$, $K(q, t)$ is obtained from $K(q, t-1)$ by adding for each of its existing subgraphs isomorphic to a $q$-clique a new vertex and joining it to all the vertices of this subgraph s〈xerting 1 for the case $q=3)$. Then, at $t=1, K(q, 1)$ resuits in the complete graph with $q+1$ vertices, $K_{q+1}$, and at $t=2$ we add $q+1$ new vertices each of them connected to all the vertices of one of the $q$ cliques $K_{q}$ 


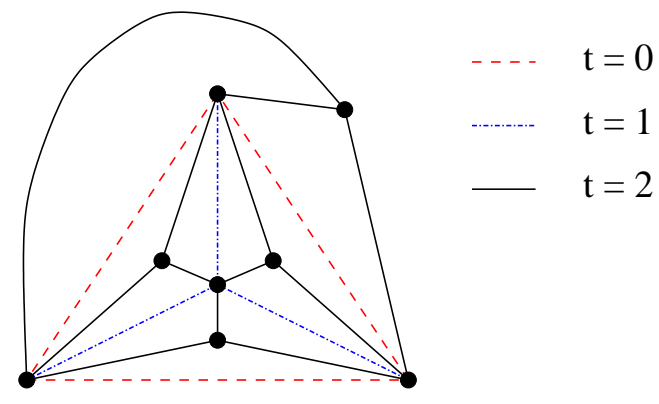

FIG. 1: (Color online) First stages of a growing recursive clique tree $K(3, t) . K(3,0)$ is the complete graph or clique $K_{3}$ (the triangle with thick edges). At each step a vertex is connected to each of existing $K_{3}$. Therefore at $t=1$ only one vertex is added, but at $t=2$, four new vertices should be connected to the four different cliques $K_{3}$.
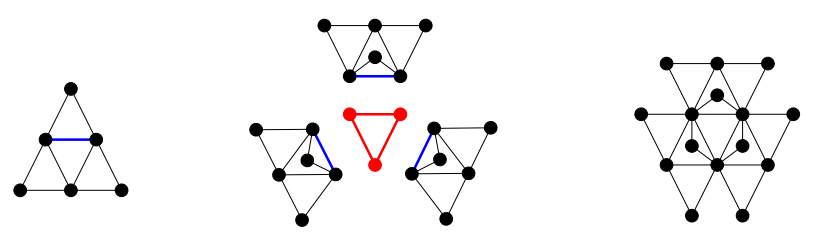

FIG. 2: (Color online) Recursive construction of $K(2, t)$. We glue a native (or initial) clique of a $K(2, t-1)$ on each 2-clique of $K_{3}$ to construct $K(2, t)$. In this figure we obtain $K(2,3)$ from three copies of $K(2,2)$ as follows: The graph at the left is $K(2,2)$ with its native 2 -clique (the thick edge). Three copies of $K(2,2)$ are glued by their natives 2 -cliques to each of the three 2 -cliques of $K_{3}$ (center) resulting in $K(2,3)$ (right).

(subgraphs of $K_{q+1}$ ), and so on.

This algorithm produces a complex growing graph with a tunable parameter $q$ which controls all its relevant characteristics. In the particular case $q=2$ we obtain the same graph as in Ref. 11]. However, our family is infinite as $q$ can take any natural value starting from 2 .

Notice that although we call the graph a recursive clique tree, the graph contains numerous cycles and hence is not a tree in the strict sense. Recursive clique-trees $K(q, t)$ are a natural generalization of trees (if one considers the case $q=1$, then we obtain a subfamily of the "usual" trees). Similar constructions in which new vertices are joined to every vertex of a given $q$ clique have been considered, in another context, in Ref. [18] and termed " $k$ trees."

Recursive construction of $K(q, t)$. There exists another interesting way to construct $K(q, t)$, which clearly shows the recursive structure of such networks. We call native clique of $K(q, t)$ its initial $q$ clique at $t=0$. Then $K(q, t)$ is constructed as follows:

At step 0 , we have the native clique $K_{q}$. For any step $t \geq 1, K(q, t)$ is constructed as follows: Consider a $(q+$ 1) clique, then every subgraph of it isomorphic to a $q$ clique (there are $q+1$ such cliques) is a native clique of a $K(q, t-1)$; see Fig. 2

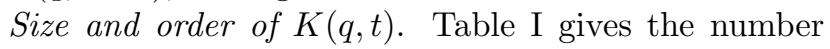

TABLE I: Number of new edges added to the recursive clique tree $K(q, t)$ at each step $t$ and total number of complete graphs $K_{q}$ at this step.

\begin{tabular}{lll}
\hline \hline Step $(t)$ & New edges & Number of $K_{q}$ \\
\hline 0 & $\frac{q(q-1)}{2}$ & 1 \\
1 & $q$ & $q+1$ \\
2 & $q(q+1)$ & $q(q+1)+(q+1)=(q+1)^{2}$ \\
3 & $q(q+1)^{2}$ & $q(q+1)^{2}+(q+1)^{2}=(q+1)^{3}$ \\
$\cdots$ & $\cdots$ & $\cdots$ \\
$\mathrm{i}$ & $q(q+1)^{i-1}$ & $(q+1)^{i}$ \\
$\mathrm{i}+1$ & $q(q+1)^{i}$ & $q(q+1)^{i}+(q+1)^{i}=(q+1)^{i+1}$ \\
$\cdots$ & $\cdots$ & $\cdots$ \\
\hline \hline
\end{tabular}

TABLE II: Distribution of vertices and degrees for the recursive clique tree $K(q, t)$ at each step $t$.

\begin{tabular}{lll}
\hline \hline Step $(t)$ & Num. vertices & Degree \\
\hline 1 & $q+1$ & $q$ \\
2 & $q+1$ & $2 q$ \\
& $q+1$ & $q$ \\
3 & $q+1$ & $q^{2}+2 q$ \\
& $q+1$ & $2 q$ \\
& $(q+1)^{2}$ & $q$ \\
4 & $q+1$ & $q^{3}+q^{2}+2 q$ \\
& $q+1$ & $q^{2}+2 q$ \\
& $(q+1)^{2}$ & $2 q$ \\
$\ldots$ & $(q+1)^{3}$ & $q$ \\
$\mathrm{i}$ & $\cdots$ & $\cdots$ \\
& $q+1$ & $q^{i-1}+q^{i-2}+\cdots+q^{2}+2 q$ \\
& $q+1$ & $q^{i-2}+\cdots+q^{2}+2 q$ \\
& $\cdots$ & $2 q$ \\
$\ldots$ & $(q+1)^{i-2}$ & $q$ \\
\hline \hline
\end{tabular}

of new edges added to the tree at each step and the total number of $K_{q}$ at this step. Therefore we can easily compute the total of edges at step $t$,

$|E|_{t}=\frac{q(q-1)}{2}+q \sum_{i=0}^{t-1}(q+1)^{i}=\frac{q(q-1)}{2}+(q+1)^{t}-1$

The distribution of vertices and degrees is given in Table III

The maximum degree at step $i$ is

$$
\Delta_{i}=\frac{q-q^{i}}{1-q}+q=\frac{q^{i}-1}{q-1}+q-1
$$

Adding up the number of vertices gives the result

$$
N_{t}=\sum_{j=1}^{t}(q+1)^{j}+(q+1)=\frac{(q+1)^{t}-1}{q}+q
$$

The average degree is then

$$
\bar{k}_{t}=\frac{2|E|_{t}}{N_{t}}=\frac{q\left[q^{2}-q+2(q+1)^{t}-2\right]}{(q+1)^{t}-1+q^{2}}
$$


[for $q=2$, it is $4 /\left(1+3^{1-t}\right)$ ]

Degree distribution. The degree spectrum of the graph is discrete: at time $t$, the number $N(k, t)$ of vertices of degree $k=q, 2 q, q^{2}+2 q, q^{3}+q^{2}+2 q, \ldots, q^{t-2}+q^{t-3}+\cdots+$ $q^{2}+2 q, q^{t-1}+q^{t-2}+\cdots+q^{2}+2 q$ is equal to $(q+1)^{t-1},(q+$ $1)^{t-2},(q+1)^{t-3},(q+1)^{t-4}, \ldots,(q+1),(q+1)$, respectively. Other values of the degree are absent. Clearly, for the large network, $N(k, t)$ decreases as a power of $k$, so the network can be called "scale free." Spaces between degrees of the spectrum grow with increasing $k$. Therefore, to relate the exponent of this discrete degree distribution to the standard $\gamma$ exponent of a continuous degree distribution for random scale free networks, we use a cumulative distribution $P_{\text {cum }}(k) \equiv \sum_{k^{\prime} \geq k} N\left(k^{\prime}, t\right) / N_{t} \sim k^{1-\gamma}$.

Here $k$ and $k^{\prime}$ are points of the discrete degree spectrum. For a degree

$$
k=q^{t-l}+q^{t-l-1}+\cdots+q+q=q\left(\frac{q^{t-l}-1}{q-1}+1\right)
$$

there are $(q+1)^{l-1}$ vertices with this exact degree.

We count now how many vertices have this and a higher degree. From the distribution

$$
\sum_{k^{\prime} \geq k} N\left(k^{\prime}, t\right)=\sum_{p=1}^{l-1}(q+1)^{p}+(q+1)=\frac{(q+1)^{l}-1}{q}+q
$$

As the total number of vertices at step $t$ is given in Eq. (3) we have

$$
\left[q\left(\frac{q^{t-l}-1}{q-1}+1\right)\right]^{1-\gamma}=\frac{\frac{(q+1)^{l}-1}{q}+q}{\frac{(q+1)^{t}-1}{q}+q}=\frac{(q+1)^{l}-1+q^{2}}{(q+1)^{t}-1+q^{2}}
$$

Therefore, for $t$ large

$$
\left(q^{t-l}\right)^{1-\gamma}=(q+1)^{l-t}
$$

and

$$
\gamma \approx 1+\frac{\ln (q+1)}{\ln q}
$$

so that $2<\gamma<2.58496$.

Also, notice that when $t$ gets large, the maximal degree of a vertex is roughly $q^{t-1} \sim N_{t}^{\ln q / \ln (q+1)}=N_{t}^{1 /(\gamma-1)}$.

Clustering distribution. The clustering coefficient $C(x)$ of a vertex $x$ is the ratio of the total number of existing connections between all $k$ its nearest neighbors and $k(k-1) / 2$, the number of all possible connections between them. The clustering of the graph is obtained averaging over all its vertices. In what follows $\mathcal{N}_{i}(x)$ will denote the total number of neighbors which $x$ has at step $i, \mathcal{K}_{i}(x)$ will denote the number of different $q$ cliques which contain $x$ at step $i$, and $\mathcal{E}_{i}(x)$ will denote the total number of edges among the neighbors of vertex $x$ also at step $i$.

Next we compute the clustering parameter for a vertex $x$ at any step of iteration.

Step 1. As $x$ belongs to $K_{q+1}$, i.e., the recursive clique tree $K(q, t)$ at step 1 , it is adjacent to $q$ other vertices $\left[\mathcal{N}_{1}(x)=q\right]$ which form a $K_{q}$. Therefore the number of edges among all vertices adjacent to $x$ is $\mathcal{E}_{1}(x)=q(q-1) / 2$. Moreover, $x$ belongs to $q$ different $q$ cliques, i.e., $\mathcal{K}_{1}(x)=q$.

Step 2. As the number of $q$ cliques in $K_{q+1}$ is $q+1$, we add this number of new vertices to the construction. But of these, $q$ will be forming -each- a different $(q+1)$ clique with $x$. Therefore $\mathcal{N}_{2}(x)=2 q$ and $\mathcal{E}_{2}(x)=q(q-1) / 2+$ $q(q-1)$. Now $x$ will belong to $\mathcal{K}_{2}(x)=\mathcal{K}_{1}(x)+q(q-1)=$ $q^{2}$ different $q$ cliques.

Step 3. We add now to the construction $\mathcal{K}_{2}(x)$ new vertices which will form each a $(q+1)$ clique with $x$. $\mathcal{E}_{3}(x)=\mathcal{E}_{2}(x)+\mathcal{K}_{2}(x)(q-1)=q(q-1) / 2+q(q-1)+$ $q^{2}(q-1) . \mathcal{K}_{3}(x)=\mathcal{K}_{2}(x)+\mathcal{K}_{2}(x)(q-1)=q^{2}+q^{2}(q-1)=$ $q^{3}$ and $\mathcal{N}_{3}(x)=\left(q^{3}-1\right) /(q-1)+q-1$.

Step $t$. We add $\mathcal{K}_{t-1}(x)$ new vertices forming each a $(q+1)$ clique with $x . \mathcal{E}_{t}(x)=\mathcal{E}_{t-1}(x)+\mathcal{K}_{t-1}(x)(q-1)=$ $q(q-1) / 2+(q-1) \sum_{i=1}^{t-1} q^{i}=(q-1)\left[q / 2+q^{t}-1 /(q-1)-\right.$ 1]. $\mathcal{K}_{t}(x)=\mathcal{K}_{t-1}(x)+\mathcal{K}_{t-1}(x)(q-1)=\mathcal{K}_{t-1}(x) q=q^{t}$ and $\mathcal{N}_{t}(x)=\left(q^{t}-1\right) /(q-1)+q-1$.

Therefore the clustering for vertex $x$ after $t$ iterations is

$$
C(x)=\frac{2 \mathcal{E}_{t}(x)}{\mathcal{N}_{t}(x)\left[\mathcal{N}_{t}(x)-1\right]}
$$

Notice that $\mathcal{N}_{t}(x)=\Delta_{t}$ is precisely the maximum degree at step $t$.

We see easily that there is a one-to-one correspondence between the degree of a vertex and its clustering. In general, we will have that for a vertex $v$ of degree $k$ the clustering parameter will be

$$
C(v)=\frac{2(q-1)\left(k-\frac{q}{2}\right)}{k(k-1)}
$$

Notice that for $q=2$ we have $C(v)=2 / k$ as in Ref. 11]. Using this result, we can compute now the clustering of the graph,

$\bar{C}_{t}=\frac{2(q+1)(q-1)\left(\Delta_{t}-\frac{q}{2}\right)}{\Delta_{t}\left(\Delta_{t}-1\right)}+\sum_{i=1}^{t-1} \frac{2(q+1)^{t-i}(q-1)\left(\Delta_{i}-\frac{q}{2}\right)}{\Delta_{i}\left(\Delta_{i}-1\right)}$

For $t \geq 7$ and for any $q \geq 3$ we have

$$
\bar{C}_{t} \geq \frac{3 q-2}{3 q-1}
$$

Thus the clustering is high, and, similarly to the $\gamma$ coefficient of the power law, is tunable by choosing the right value of $q$ : in particular, $\bar{C}$ ranges from $\frac{4}{5}$ (in the case $q=2$ ) to a limit of 1 when $q$ gets large.

Diameter. We recall that the diameter of a graph is the maximum of all distances between any two vertices of the graph. Computing the exact diameter of $K(q, t)$ can be done analytically, and gives the result shown below. Here, we present the main ideas of this analysis.

First, we note that for any step $t$, the diameter always lies between pair of vertices that have just been created at 
this step. We will call such vertices outervertices. Then, we note that, by construction, no two outervertices created at the same step $t$ can be connected. Hence, if $v_{t}$ is an outervertex created at step $t$, then $v_{t}$ has been connected to a $q$ clique composed of vertices created at pairwise different steps $t_{1}<t_{2}<\cdots<t_{q}$ and consequently, $t_{1} \leq t-q$. Now we want to know the distance between two outervertices $u_{t}$ and $v_{t}$. The idea is, starting from $u_{t}$, to "get back" to $K(q, 1)$ by jumps from $u_{t}$ to $u_{t-q}$, then to $u_{t-2 q}$, etc. Hence it takes at most about $t / q$ jumps to go from $u_{t}$ to $K(q, 1)$. Then it takes at most as many jumps to go from $K(q, 1)$ to $v_{t}$ and we conclude that the diameter cannot be bigger than (roughly) $2 t / q$.

More precisely, the exact formula for the diameter of $K(q, t)$, denoted $\operatorname{Diam}[K(q, t)]$, is the following :

$$
\operatorname{Diam}[K(q, t)]=2\left(\left\lfloor\frac{t-2}{q}\right\rfloor+1\right)+f(q, t)
$$

where $f(q, t)=0$ if $t-\lfloor(t-2) / q\rfloor q \leq\lceil(q+1) / 2\rceil$, and 1 otherwise. This value can be obtained by a sharp analytical proof.

When $t$ gets large, then $\operatorname{Diam}[K(q, t)] \sim 2 t / q$, while $N_{t} \sim q^{t-1}$, thus the diameter clearly grows logarithmically with the number of vertices.

Discussion. The recursive clique-trees $K(q, t)$ which we study in this paper are recursively constructed graphs which have both small-world and scale-free characteristics. By choosing adequately the value of $q$ it is possible to obtain different clustering parameters and power-law exponents (the clustering of the graph ranges from 0.8 to 1 while the power-law exponent takes values between 2 and 2.58496). It has been shown that many networks modeling complex systems such as the WWW, Internet, movie actors, the Erdős number, interactions in protein complexes of Saccharomyces cerevisae, the metabolic network of Escherichia coli, etc., have clustering and powerlaw parameters precisely in these ranges, see, for example, Refs. [2, 6, 15, 19, 20? ]. Moreover, the recursive clique trees are actually a deterministic tunable generalization of the scale-free growing networks introduced in Ref. 21] in which one vertex is created per unit time and connects to both the ends of a randomly chosen edge. These networks were further generalized and called "pseudofractal" graph in [11], and correspond to the particular case $q=2$ of the recursive clique trees. Variations of our construction, for example, by choosing that in different steps the new vertices added are attached to cliques of different size, would allow a richer structure and more flexibility in the control of the clustering and power-law exponent and other relevant network parameters.

The authors thank a Picasso bilateral grant, references HF2001-0056 (Spain) and 04295SG (France). Additional support for the first author was provided by the Ministry of Science and Technology, Spain, and the European Regional Development Fund (ERDF) under Project No. TIC2002-00155.
[1] A.-L. Barabási and R. Albert, Science 286, 509 (1999).

[2] D.J. Watts and H. Strogatz, Nature (London) 393, 440 (1998).

[3] L. Adamic and B. Huberman, Science 287, 2115 (2000).

[4] R. Albert, H. Jeong, and A.-L. Barabási, Nature (London) 401, 130 (1999).

[5] E. Almaas, R.V. Kulkarni, and D. Stroud, Phys. Rev. Lett. 88, 098101 (2002).

[6] M. Faloutsos, P. Faloutsos, and C. Faloutsos, Comput. Commun. Rev. 29, 251 (1999).

[7] R. Albert and A.-L. Barabási, Rev. Mod. Phys. 74, 47 (2002).

[8] F. Comellas, J. Ozón, and J.G. Peters, Inf. Process. Lett. 76, 83 (2000).

[9] F. Comellas, and M. Sampels, Physica A 309, 231 (2002).

[10] A.-L. Barabási, E. Ravasz, and T. Vicsek, Physica A 299, 559 (2001).

[11] S.N. Dorogovtsev, A.V. Goltsev, and J.F.F. Mendes, Phys. Rev. E 65, 066122 (2002).

[12] E. Ravasz and A.-L. Barabási, Phys. Rev. E 67, 026112 (2003).
[13] J.D. Noh, Phys. Rev. E 67, 045103 (2003).

[14] S. Jung, S. Kim, and B. Kahng, Phys. Rev. E 65, 056101 (2002).

[15] J.F.F. Mendes, in Statistical Mechanics of Complex Networks, edited by R. Pastor-Satorras, M. Rubi, and A. Diaz-Guilera, Lecture Notes in Physics Vol. 625 (Springer, Berlin, 2003), pp. 88-113.

[16] S.L. Tauro, C. Palmer, G. Siganos, and M. Faloutsos, in Global Telecommunications Conference GLOBECOM '01 (IEEE, Piscataway, NJ, 2001), Vol 3, pp. 1667 -1671.

[17] D.B. West, Introduction to Graph Theory (Prentice-Hall, Upper Saddle River, NJ, 2001).

[18] H.L. Bodlaender, Bull. EATCS 36, 116 (1988).

[19] H. Jeong, B. Tombor, R. Albert, Z. N. Oltvai, and A.-L. Barabási, Nature (London) 407, 651 (2000).

[20] K.I. Goh, E.S. Oh, H. Jeong, B. Kahng, and D. Kim, Proc. Natl. Acad. Sci. U.S.A. 99, 12583 (2002).

[21] S.N. Dorogovtsev, J.F.F. Mendes, and A.N. Samukhin, Phys. Rev. E 63, 062101 (2001). 